\title{
Fibrin-thrombin sealant does not reduce lymphocele formation in patients with inguinofemoral lymphadenectomy for vulvar
} cancer

This article was published in the following Dove Press journal:

Cancer Management and Research

\author{
Flurina AM Saner (D) \\ Andreas Schötzau (iD ${ }^{2}$ \\ Gillian Mackay (D) \\ Viola Heinzelmann- \\ Schwarz (iD) 1,2 \\ Céline Montavon Sartorius (iD) \\ 'Department of Gynecology and \\ Gynecological Oncology, Hospital for \\ Women, University Hospital Basel and \\ University of Basel, Basel, Switzerland; \\ ${ }^{2}$ Ovarian Cancer Research, Department \\ of Biomedicine, University Hospital Basel \\ and University of Basel, Basel, \\ Switzerland
}

Correspondence: Céline Montavon Sartorius

Department of Gynecology and Gynecological Oncology, Hospital for

Women, University Hospital Basel, Spitalstrasse 21, Basel 403I, Switzerland

Tel +4 I6I 2659099

Fax +4 161 2659199

Email celine.montavon@usb.ch
Purpose: Inguinofemoral lymphadenectomy (IFLD) is associated with very high morbidity. Fibrin sealant patches are used in various surgical procedures to prevent hematomas and lymphoceles. Here, we report a single-institution experience of fibrin sealants after IFLD in vulvar cancer and give an overview of the current literature.

Patients and methods: We retrospectively analyzed outcome data on vulvar cancer patients who underwent bilateral IFLD between November 2014 and June 2016 at the University Hospital Basel. A fibrin sealant patch $\left(\right.$ Tachosil $^{\mathbb{R}}$ ) was placed in one groin only. Postsurgical fluid collection and complications were compared between both groins; time courses of lymphocele formation were analyzed using a linear mixed-effects regression model.

Results: Postsurgical outcome of 11 consecutive patients with bilateral IFLD for vulvar cancer was assessed for a median follow-up of 32 days (range 12-77). Significantly larger lymphoceles were detected over time in the groin with the fibrin sealant patch (median $20 \mathrm{~mL}$ vs $5 \mathrm{~mL}$ without patch, $p=0.002$ ), and more punctures for symptomatic lymphocele were required on this side. The infection rate did not differ between sides.

Conclusion: Fibrin sealant patches should not routinely be used for prevention of lymphoceles after IFLD for vulvar cancer. They do not seem to reduce lymph collection, the need for puncture or the infection risk after lymphadenectomy in our experience and according to a general literature review.

Keywords: lymphedema, lymphovascular leakage, gynecological malignancy, fibrin sealant, TachoSil $^{\circledR}$

\section{Introduction}

Vulvar cancer is a rare gynecological disease, with an incidence of 2-3/100,000 women; it typically affects patients of advanced age. Much has been done in the past to try to reduce complications following vulvar cancer surgery, which consists of resection of the vulvar tumor and groin staging and/or dissection. Morbidity is very high, often leading to several weeks of hospitalization, which may postpone adjuvant therapy.

However, surgery remains the standard treatment of locoregional disease. Compared to en bloc resection and radical vulvectomy with bilateral inguinofemoral lymphadenectomy (IFLD), radical local excision and inguinofemoral lymph 
node staging and/or dissection are associated with a reduced morbidity whilst preserving oncogenic safety. It is clearly being favored in early-stage vulvar carcinoma and is now the standard of care. Additional therapeutic approaches consist of radiotherapy and combined chemoradiation, but evidence is scarce. These are valuable options in primary unresectable tumors and in the neoadjuvant setting to achieve resectability in cases where an exenteration might be the only alternative. ${ }^{1}$

Lymph node status is the single most important prognostic factor in vulvar cancer. In the absence of lymphatic metastases, 5 -year survival can reach up to $84-96 \%$, whereas it decreases to $30 \%$ or lower in patients with positive inguinofemoral lymph nodes. ${ }^{2,3}$ Given the impact of lymphatic metastases on future prognosis, assessment of the nodal status is crucial in vulvar cancer patients. According to current guidelines, removal of lymphatic tissue is dispensable only in International Federation of Gynecology and Obstetrics (FIGO) stage IA cases (tumor size $\leq 2 \mathrm{~cm}$, vulvar or perineal location, $\leq 1 \mathrm{~mm}$ depth of invasion). The vast majority of patients require inguinofemoral lymph node staging.

IFLD is associated with very high morbidity. Lymphedema of the leg, lymphocele with repeated punctures, wound breakdown and infections account for the most common complications, affecting $50-85 \%$ of patients with IFLD. ${ }^{4,5}$

Sentinel node biopsy currently represents the best attempt to reduce the morbidity of groin dissection, but is recommended in selected patients only. In approximately half of the patients, IFLD is still indicated; therefore, further strategies to reduce morbidity need to be explored. ${ }^{4}$ The use of a fibrin sealant patch was effective in improving hemostasis after surgery on the liver and kidney and has shown promising results in reducing fluid collection after mediastinal, ${ }^{6}$ axillary $^{7}$ and pelvic lymphadenectomy. ${ }^{8,9}$

These findings led us to investigate our own institutional practice, which occasionally included the use of a fibrin sealant in order to generate data on its effectivity as part of quality control. A true clinical benefit would justify integration of this procedure as standard of care in our surgical approach to IFLD.

The hypothesis behind the use of fibrin sealants is that they could occlude the superficial and deep fascia, hereby potentially reducing the volume of lymph accumulation and lymphocele formation in patients affected with vulvar cancer requiring bilateral IFLD. However, after using the fibrin sealant patch on very few patients, there was clinical doubt about its benefit, and therefore statistical analysis of the follow-up data was undertaken. This study investigates if fibrin sealants prevent lymphocele formation and secondary complications after IFLD in vulvar cancer. We additionally performed a literature review in order to put our data into context.

\section{Patients and methods}

We performed a retrospective data analysis on a total of 11 consecutive patients who underwent bilateral IFLD for vulvar cancer at the Hospital for Women, University Hospital Basel between November 2014 and June 2016. Standard groin dissection was performed as previously described. ${ }^{10}$ A single surgical team including a gynecologic oncologist and a gynecological oncology fellow performed the surgeries in a consistent manner. Immediately after the IFLD and before closing the wound, a TachoSil ${ }^{\circledR}$ patch of $9.5 \mathrm{~cm} \times 4.8 \mathrm{~cm} \times 0.5 \mathrm{~cm}$ in size (human thrombin/human fibrinogen, Takeda, Freienbach, Switzerland) was placed in one groin. The operating room nurse was asked to allocate the side that the fibrin sealant should be inserted. The patch, containing a fixed dosage of fibrinogen $5.5 \mathrm{mg} / \mathrm{cm}^{2}$ and thrombin $2.0 \mathrm{IU} / \mathrm{cm}^{2}$ on its yellow active side, was applied as recommended by the manufacturer. Its medical use is permitted by the Swiss Agency for Therapeutic Products (Swissmedic, $\mathrm{n}^{\circ} 00670$ ). No wound drain was placed. A second-generation cephalosporin antibiotic (cefuroxime 1.5 $\mathrm{g}$ intravenously) was administered perioperatively as a single dose for infection prophylaxis. There was no standard postoperative antibiotic treatment. A weight adapted postoperative thrombosis prophylaxis was administered with subcutaneous low-molecular-weight heparin (dalteparin).

The total amount of accumulated lymph fluid (primary endpoint) was either documented by ultrasound or collected by drain, puncture or evacuation for both groins every other day in-hospital and during outpatient followup or emergency consultations. Drains were used as clinically indicated for increased intraoperative bleeding tendency only; otherwise, this procedure was not routine. The drains were removed after the fluid production decreased to $<30 \mathrm{~mL}$ per day. Lymphocele was only punctured in symptomatic patients. As long as the wound was open, the lymph fluid was manually evacuated and collected in a container for measurement.

Complications (secondary endpoint) including punctures for symptomatic lymphocele, local infections requiring antibiotic therapy, re-admission to hospital and wound revision were recorded as part of our usual oncological follow-up. 
This study was approved by the Swiss Medical Ethics Committee, the Ethikkommission Nordwest- und Zentralschweiz (EKNZ project ID 2018-01701). Patients were consented for analysis of their de-identified data for research. This retrospective observational study carried no risk of harm for patients, and their data were used anonymously and patient confidentiality was maintained throughout.

\section{Statistical analysis}

Descriptive statistics were summarized using mean, median and range or counts and percentages as appropriate. $P$-values were estimated using independent $t$-test for means, MannWhitney U-test for medians or Fisher's exact test in case of counts and percentages.

The total amount of lymphocele in the groin with and the groin without a fibrin sealant patch was analyzed. Before evaluation, lymphocele was square root (sqrt) transformed to achieve approximate normal distribution. The time courses of fluid collection in the groin with and without the fibrin sealant were compared using a linear mixed-effects model with days and patch as continuous predictors. Subject was treated as a random factor. Mixed-effects models were suitable tools for repeated measure data and accounted for correlations within each subject. A (nonparametric) Wilcoxon signed rank test was also performed to compare the median amount of fluid between study groups (fibrin sealant versus no sealant).

Results are presented as regression slopes of the predictors with corresponding $95 \% \mathrm{CI}$ and $p$-values. For continuous predictors (days), the slope indicates the increase per day; for a categorical predictor such as the fibrin patch, it indicates the difference of the means.

A $p$-value $<0.05$ is considered as significant. All evaluations were performed by a statistician using the current version of the statistical software R.

\section{Results}

We analyzed and compared postoperative data of 11 patients with an average age of 72 years (range 62-83). With the exception of one patient with vulvar melanoma, all patients had a squamous cell carcinoma of the vulva, most commonly FIGO stage IB $(55 \%, n=6)$ and grade $3(50 \%, n=5)$. One patient with a multifocal grade 3 squamous cell carcinoma involving the midline and considered as early stage on the pathology report underwent a bilateral IFLD as she was clinically clearly staged as FIGO IB. The depth of invasion could not be measured precisely in the initial biopsy because of the fragmented nature of the tissue. The tumor measured $0.2 \times 0.9 \mathrm{~mm}$ in one block including epidermis, but additional smaller pieces of the same biopsy derived from deeper layers also contained carcinoma, adding up to an infiltration depth of $>1 \mathrm{~mm}$, resulting in the need to explore the lymph node status. A drain was used in two patients in the groin without fibrin sealant because of an increased intraoperative bleeding tendency. For one patient, the drain was removed after 4 days (fluid $<30 \mathrm{~mL}$ ); however, she developed a symptomatic lymphocele requiring sequential punctures after 19 days on the side of the fibrin patch. The second patient had her drain removed after 12 days and did not show any complication. Another patient had bilateral drains placed on postoperative day 27 due to bilateral wound revision required for poor healing and infection and was subsequently excluded from the study. All patient characteristics are summarized in Table 1.

Overall, lymph nodes were positive in three patients; two patients had bilateral metastatic disease. The number of lymph nodes removed (mean 7.0 vs $7.8, p=0.52$ ) and the proportion of positive nodes (mean $2.6 \%$ vs $5.8 \%, p=0.45$ ) did not differ between the fibrin sealant and control side, respectively.

Postoperative course was documented for a median of 32 days (range 12-77). Of the overall 207 observations, $45.4 \%$ (94/207) consisted of actual fluid collection either by drain, puncture, or evacuation. Larger lymphoceles were detected in the groin with the fibrinogen patch with a median

\section{Table I Patient characteristics}

\begin{tabular}{|c|l|l|}
\hline Total & n= I I & I00\% \\
\hline FIGO & & \\
IB & $6 *$ & $55 \%$ \\
II & 2 & $18 \%$ \\
IIIA & 1 & $9 \%$ \\
IIIB & 0 & $0 \%$ \\
IIIC & 1 & $9 \%$ \\
IVA & 1 & $9 \%$ \\
\hline Grading & & \\
GI & 1 & $10 \%$ \\
G2 & 4 & $40 \%$ \\
G3 & 5 & $50 \%$ \\
\hline Histology & & $91 \%$ \\
SCC & 10 & $9 \%$ \\
Melanoma & 1 & Range: 62-83 \\
\hline Median age (years) & 72 & Range: 12-77 \\
\hline Median follow-up (days) & 32 & \\
\hline
\end{tabular}

Note: *One patient included with early-stage vulvar carcinoma according to the original pathology report but with fragmented biopsies and a total depth of invasion of $>$ I mm (clinically FIGO stage IB).

Abbreviation: FIGO, nternational Federation of Gynecology and Obstetrics; SCC, squamous cell carcinoma. 
Table 2 Surgical characteristics and postoperative groin complications for the fibrinogen patch versus control side in II patients with bilateral inguinofemoral lymphadenectomy for vulvar cancer

\begin{tabular}{|l|l|l|l|}
\hline & $\begin{array}{l}\text { Fibrin } \\
\text { patch } \\
\text { side }\end{array}$ & $\begin{array}{l}\text { Control } \\
\text { side }\end{array}$ & $\boldsymbol{p}$ \\
\hline $\begin{array}{l}\text { Lymph nodes removed, } \\
\text { mean } \\
\text { Lymph nodes with } \\
\text { metastasis }\end{array}$ & 7.0 & 7.8 & 0.52 \\
$\begin{array}{l}\text { Fluid Volume (mL), median } \\
\text { (range) }\end{array}$ & $\begin{array}{l}2 / 77 \\
\text { Patients with } \\
\text { complications (n=I I) }\end{array}$ & $5 / 86(5.8 \%)$ & 0.45 \\
$\begin{array}{l}\text { Puncture/fluid evacuation } \\
\text { Recurrent puncture }>\text { I4 days } \\
\text { Infections (antibiotics) }\end{array}$ & $5(0-535)$ & $\mathbf{0 . 0 0 2}$ \\
\hline
\end{tabular}

Notes: Complications are indicated as the number of evacuative punctures, number of groins requiring repetitive punctures for a period longer than 14 days post surgery and number of wound infections treated with systemic antibiotic therapy. Bold values indicate statistical significance.

of $20 \mathrm{~mL}$ fluid (range $0-300$ ) versus $5 \mathrm{~mL}$ (range $0-535$ ) on the control side (Table 2).

The correlation between the use of fibrin sealant patch and the amount of fluid collection over time was analyzed in a linear mixed-effects model. The patch showed a significantly negative effect on the fluid collection with an increasing amount over time (slope on original scale $=35.03$, $\mathrm{CI}=12.51$ to $57.55, p=0.002$; Figure 1). In order to compare the amount of fluid between both groins, we also performed a nonparametric test on median values. The Wilcoxon signed rank test was not significant $(p=0.086)$, but showed a trend pointing into the same direction as the parametric mixedeffects model, which compares mean values ( $p=0.002)$.

Postoperative complications did not differ significantly between the two groups. Ten of 11 patients $(91 \%)$ required a single or serial evacuative punctures for symptomatic lymphoceles. In eight patients, a bilateral puncture was undertaken, whereas two patients had to be punctured only in the groin containing the fibrin sealant. After 14 days, $82 \%(n=9 / 11)$ of the groins containing the sealant still required a puncture, compared to $45 \%(n=5 / 11)$ of the control side $(p=0.18)$. On the side of fibrin sealant, more punctures or evacuations $(\mathrm{n}=52)$ were required, and a cumulative volume of $5,445 \mathrm{~mL}$ of fluid was collected. In contrast, on the side without sealant, 30 punctures were needed and 3,152 $\mathrm{mL}$ drained in total. After 2 weeks, more punctures were still required on the fibrin sealant side, with a total of 26 punctures for the drainage of overall

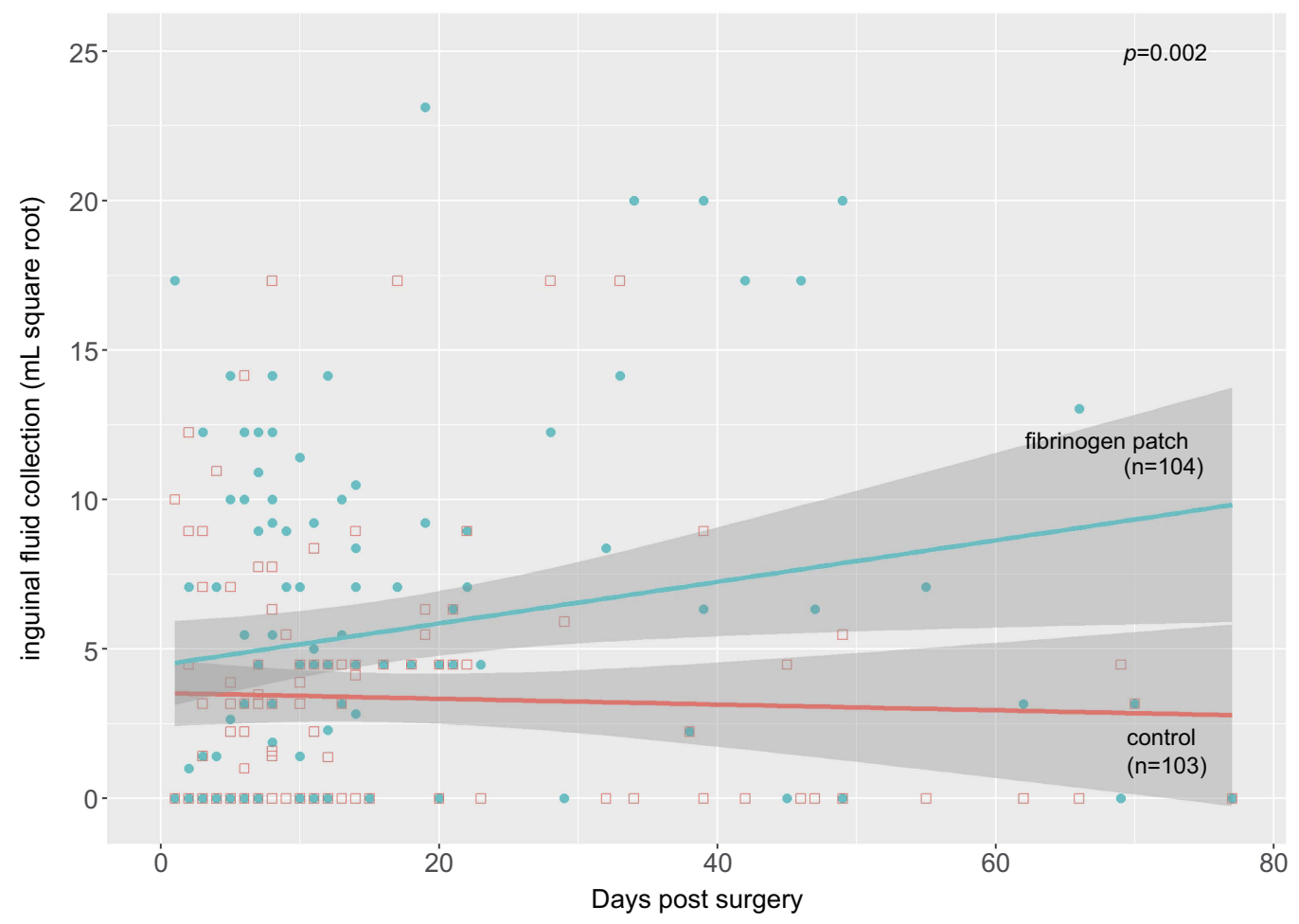

Figure I Scatter plot showing the time course of fluid collection in the groin containing a fibrin sealant (turquoise) and in the groin with no sealant patch (red). Each dot represents a measure $(n=207)$ of the fluid amount in $\mathrm{mL}$ at a specific time point. Fluid is displayed on square root scale. 
3,100 mL compared to 18 punctures for $1,455 \mathrm{~mL}$ on the control side. An oral antibiotic course was administered to three patients $(27.3 \%)$ suffering from wound infections or infected lymphoceles. The infection rate was similar for both sides (one wound infection was bilateral, one was on the side with the sealant patch and one was on the side without it). Other complications related to the use of a thrombin/fibrinogen patch such as thromboembolic events or allergic reactions were not recorded. The secondary endpoint results are shown in Table 2.

\section{Discussion}

The use of a fibrin sealant patch in bilateral IFLD for vulvar cancer showed in our experience a significant increase of lymphocele volume over time after inguinal application. The purpose of this analysis was an institutional quality evaluation in order to include the fibrin sealant in the surgical standard.

However, we stopped using the fibrin sealant as a routine procedure after only 11 patients because of clinical doubt as to its benefit. The statistical results confirmed the clinical impression; the use of the fibrinogen patch to reduce the groin lymphocele formation failed in our cohort. On the contrary, we observed a significantly larger fluid collection over time in the groin when the patch was applied $(p=0.002)$ and more punctures or fluid evacuations on this side. Differences of the median volume between the sealant and the control side in a nonparametric test pointed into the same direction.

The central etiology of postoperative lymphoceles is thought to be due to oozing of injured small lymph and blood vessels as well as inflammatory exudation after tissue damage. ${ }^{11}$ A prolonged hospital stay, wound breakdown, infection and delay of adjuvant therapy are the most frequent sequelae of lymphoceles after groin dissection and significantly impact the quality of life for these patients. $^{12}$ Identification of women at risk and the use of effective prevention methods are important to reduce the risk of lymphocele formation.

Higher age, diabetes and "en bloc" surgery were risk factors for any short-term complication in a large retrospective study on 164 patients with IFLD for vulvar cancer, but no risk factor for the formation of lymphocele was identified in this trial. ${ }^{4}$ Interestingly, in contrast to other studies, a higher number of resected lymph nodes were found to be protective for any long-term complications, whereas younger patients and patients with postoperative lymphocele showed an increased risk of developing longterm complications such as lymphedema and erysipelas.
The introduction of sentinel node biopsies in unifocal vulvar cancer with $<4 \mathrm{~cm}$ size and nonsuspicious lymph nodes on clinical and radiological examination has clearly the biggest impact in risk reduction of postoperative lymphoceles to date. ${ }^{13}$ Although the sentinel node biopsy has been accepted as a standard procedure, a critical appraisal has been newly addressed regarding its false-negative rate of $5-10 \%$, resulting in a $1 \%$ risk of death for early-stage vulvar cancer patients. ${ }^{14}$ Therefore, most patients with vulvar cancer will still undergo IFLD, which underlines the importance of finding modalities to decrease the complication rate.

Similar to fibrin sealants, routine wound drainage to prevent lymphocele formation is an area of intense debate. Besides possible benefits such as reduced morbidity and earlier mobility, a drain can cause an increased fluid secretion through irritation of the wound or increase the risk of an infection, and it remains unclear how long the drain should stay in the groin. A prospective study on IFLD in vulvar cancer clearly showed that a volume-controlled drainage, where the drain is removed once the fluid production declined to $<30 \mathrm{~mL} / 24 \mathrm{hrs}$, resulted in significantly fewer lymphocele and secondary complications compared to a routine short-term drainage for 5 days. ${ }^{15}$ In a retrospective analysis, a wound breakdown occurred significantly more often after a shortterm postsurgical drainage, and long-term usage was found to be associated with an increased risk of lymphedema. ${ }^{16}$ The complication rate also seems to vary between different drain types. ${ }^{17}$ A consensus on postsurgical drain management unfortunately has not been reached.

The preservation of the superficial (camper) and deep (cribriform) fascia reduces the rate of lymphoceles, as well as $38 \%$ of the overall short-term and $14 \%$ of the long-term complications. ${ }^{18}$ Sparing of the saphenous vein reduces postoperative wound complications but has no impact on lymphocele formation. Covering the femoral vessels either with cadaver or artificial dura mater or by transposition of the sartorius muscle does not show any protective effect in terms of lymph collection either. ${ }^{5}$ The use of ultrasonic scalpels has also failed to reduce the drainage volume after IFLD. ${ }^{19}$ Other potential preventive modalities for lymphocele include the (robotic) video endoscopic inguinal lymphadenectomy (R-)VEIL, ${ }^{20}$ FloSeal ${ }^{2}$ (a hemostatic matrix used in pelvic lymphadenectomy) and lymphatic microsurgical preventive healing approach LYMPHA. $^{21,22}$ Initial studies on LYMPHA microsurgery, which involves anastomoses of lymphatic vessels, showed promising results for preventing lymphedema after both axillary and inguinofemoral lymphadenectomies. ${ }^{22,23}$ 
Fibrin, once converted from fibrinogen by thrombin, is a polymerized plasmatic protein, which forms hemostatic clots on wounds. Commercial fibrin sealants, containing concentrated human fibrinogen and thrombin as key components, are available as solutions, sprays, fleeces, sponges or mesh. ${ }^{24,25}$ By imitating the final part of the coagulation cascade, they are designed to support hemostasis and tissue sealing during surgical procedures. Further potential benefits of their use in surgery result from a reduced blood loss, shorter operation time and a decreased risk for complications such as hematoma and seroma by sealing leaky vessels.

To date, few adverse outcomes of fibrin sealants have been reported and the majority of trials found no harm related to their use. ${ }^{26}$ Intravascular application with subsequent thrombosis or gas embolism in the case of spray usage, allergic reactions, poor wound healing and infections account for possible and reported harms of sealants. In addition, the plasma-derived nature of the human and bovine components incorporates a potential risk for transmission of blood-borne diseases. ${ }^{27}$

A meta-analysis of 32 randomized controlled trials (RCTs) investigated risks and benefits of fibrin sealants in different surgical disciplines. ${ }^{26}$ This analysis showed a nonsignificant risk reduction for seroma formation with fibrin sealants over standard treatment (OR 0.84, 95\% CI $0.68-1.04, p=0.13$ ). A significantly decreased risk for hematoma with fibrin sealants was found only for hernia surgery. For breast $(p=0.87)$, orthopedic $(p=0.20)$ and upper GI tract surgery ( $p=0.88$ ), a trend toward a reduced risk for hematoma was reported without reaching statistical significance.

Over the last decade, there has been much debate over the prevention of seroma after axillary lymphadenectomy in breast cancer surgery by using fibrin glue. The benefits of sealants are controversial; several trials show no effect on axillary seroma, ${ }^{28-30}$ whereas others reported a reduced drainage volume but without decreasing seroma formation or necessity for evacuation. ${ }^{7,31}$

Poor quality of trials was a concern of a 2013 Cochrane Intervention Review on fibrin glue in surgery for breast cancer. $^{32}$ Analysis of 18 RCTs including 1,252 patients revealed that the incidence of seroma following breast and axillary surgery was not reduced when fibrin glue was added under skin flaps (RR 1.02, 95\% CI 0.90-1.16, $p=0.73$ ).

Few trials have investigated the use of fibrin glue in vulvar cancer surgery. An observational case-control study on 49 patients (24 IFLDs with application of a fibrinogen patch and 25 IFLDs without it) found that application of a fibrin sealant patch significantly reduced postoperative lymphorrhea and prevented lymphocyst formation $(p=0.004) .{ }^{33}$ A Jackson-Pratt closed suction drain was inserted and left in place until the daily drainage volume was $\leq 50 \mathrm{~mL}$. In contrast, we did not use routine groin drainage as it has no influence on lymphocele and wound infection rate. ${ }^{16}$ Another major difference is that we compared the fluid collection between both groins within the same patient, as the fibrin sealant was applied only on one side during a bilateral IFLD.

In the only prospective randomized trial to date, the usage of VH fibrin sealant spray after IFLD in 150 patients with vulvar cancer had no impact on the incidence of lymphoceles, inguinal infection, wound breakdown or lymphedema. ${ }^{17}$ However, significantly more patients with fibrin sealant developed a vulvar infection (33\% vs $14 \%$, $p=0.0098$ ). In our cohort, the rate of postoperative infection or wound breakdown did not differ between the two groups.

Our findings are also consistent with a meta-analysis of six RCT investigating fibrin sealants in groin dissection for malignancies, where no reduction of morbidity was found after application of a sealant. ${ }^{34,35}$ In contrast, our data actually showed an increase of lymph collection in the groin where a fibrin patch was placed.

The small number of patients included in our study is a limitation to our results; however, we stopped the use of the fibrin sealant patch with intent due to doubts about its clinical benefit. The statistical results confirmed this impression as a significantly higher lymph collection in the groin containing the patch was recorded $(p=0.002)$. The comparison between the conventional treatment and fibrin sealant within the same patient reduced the interindividual bias dramatically and is clearly a strength of our study. Furthermore, the surgery was performed in a consistent manner and by the same gynecological oncologists. The mean number of lymph nodes removed (7-8 lymph nodes per side) is in line with recommendations. ${ }^{36}$ There were slightly more pathological lymph nodes removed on the control side; however, this finding did not seem to influence the results toward more lymph production.

\section{Conclusion}

Besides the lack of risk reduction for lymphoceles, infections or the need for punctures, the costs of a fibrin patch and the potential harms are additional reasons for not recommending routine use of fibrin sealants for prevention of postoperative groin lymphocele in vulvar cancer. We would not necessarily recommend further trials analyzing the use of fibrin sealant for reduction of lymphocele after IFLD, as the benefit of the fibrin patch for this indication 
appears generally unconvincing in other diseases. Larger RCTs in vulvar cancer patients are needed in order to reduce the morbidity after groin dissection. This remains a challenge due to the rarity of the disease. The development of new preventive and therapeutic strategies - such as an alternative to groin dissection - would be a great step forward, assuming an equal oncologic safety is achievable.

\section{Disclosure}

The authors report no conflicts of interest in this work.

\section{References}

1. de Hullu JA, van der Avoort IA, Oonk MH, van der Zee AG. Management of vulvar cancers. Eur J Surg Oncol. 2006;32 (8):825-831. doi:10.1016/j.ejso.2006.03.035

2. Zhou J, Shan G. The prognostic role of FIGO stage in patients with vulvar cancer: a systematic review and meta-analysis. Curr Med Res Opin. 2016;32(6):1121-1130. doi:10.1185/03007995.2016.1162147

3. Hacker NF, Berek JS, Lagasse LD, Leuchter RS, Moore JG. Management of regional lymph nodes and their prognostic influence in vulvar cancer. Obstet Gynecol. 1983;61(4):408-412.

4. Hinten F, van Den Einden LC, Hendriks JC, et al. Risk factors for short- and long-term complications after groin surgery in vulvar cancer. Br J Cancer. 2011;105(9):1279-1287. doi:10.1038/ bjc.2011.407

5. Pouwer AW, Arts HJ, van der Velden J, de Hullu JA. Limiting the morbidity of inguinofemoral lymphadenectomy in vulvar cancer patients; a review. Expert Rev Anticancer Ther. 2017;17 (7):615-624. doi:10.1080/14737140.2017.1337513

6. Czerny M, Fleck T, Salat A, et al. Sealing of the mediastinum with a local hemostyptic agent reduces chest tube duration after complete mediastinal lymph node dissection for stage I and II non-small cell lung carcinoma. Ann Thorac Surg. 2004;77(3):1028-1032. doi:10.1016/j.athoracsur.2003.08.041

7. Benevento R, Santoriello A, Pellino G, et al. The effects of low-thrombin fibrin sealant on wound serous drainage, seroma formation and length of postoperative stay in patients undergoing axillary node dissection for breast cancer. A randomized controlled trial. Int J Surg. 2014;12(11):1210-1215. doi:10.1016/j.ijsu.2014.10.005

8. Grimm C, Polterauer S, Helmy S, et al. A collagen-fibrin patch (Tachosil(R)) for the prevention of symptomatic lymphoceles after pelvic lymphadenectomy in women with gynecologic malignancies: a randomized clinical trial. BMC Cancer. 2014;14:635. doi:10.1186/ 1471-2407-14-635

9. Tinelli A, Mynbaev OA, Tsin DA, et al. Lymphocele prevention after pelvic laparoscopic lymphadenectomy by a collagen patch coated with human coagulation factors: a matched case-control study. Int $J$ Gynecol Cancer. 2013;23(5):956-963. doi:10.1097/ IGC.0b013e31828eeea4

10. Nicklin JL, Hacker NF, Heintze SW, Van Eijkeren M, Durham NJ. An anatomical study of inguinal lymph node topography and clinical implications for the surgical management of vulval cancer. Int J Gynecol Cancer. 1995;5(2):128-133.

11. Agrawal A, Ayantunde AA, Cheung KL. Concepts of seroma formation and prevention in breast cancer surgery. ANZ J Surg. 2006;76 (12):1088-1095. doi:10.1111/j.1445-2197.2006.03949.x

12. Farrell R, Gebski V, Hacker NF. Quality of life after complete lymphadenectomy for vulvar cancer: do women prefer sentinel lymph node biopsy? Int J Gynecol Cancer. 2014;24(4):813-819. doi:10.1097/IGC.0000000000000101
13. Van der Zee AG, Oonk MH, De Hullu JA, et al. Sentinel node dissection is safe in the treatment of early-stage vulvar cancer. J Clin Oncol. 2008;26(6):884-889. doi:10.1200/JCO.2007.14.0566

14. Hacker NF, Barlow EL. Sentinel node biopsy in vulvar cancer: A critical appraisal. Asian J Oncol. 2017;3:5-11. doi:10.4103/2454-6798.209328

15. Pouwer AW, Hinten F, van der Velden J, et al. Volume-controlled versus short drainage after inguinofemoral lymphadenectomy in vulvar cancer patients: A Dutch nationwide prospective study. Gynecol Oncol. 2017;146(3):580-587. doi:10.1016/j.ygyno.2017.06.031

16. Walker KF, Day H, Abu J, Nunns D, Williamson K, Duncan T. Do surgical techniques used in groin lymphadenectomy for vulval cancer affect morbidity rates? Int J Gynecol Cancer. 2011;21(8):1495-1499. doi:10.1097/IGC.0b013e318228f314

17. Carlson JW, Kauderer J, Walker JL, et al. A randomized phase III trial of VH fibrin sealant to reduce lymphedema after inguinal lymph node dissection: a Gynecologic Oncology Group study. Gynecol Oncol. 2008;110(1):76-82. doi:10.1016/j.ygyno.2008.03.005

18. Micheletti L, Bogliatto F, Massobrio M. Groin lymphadenectomy with preservation of femoral fascia: total inguinofemoral node dissection for treatment of vulvar carcinoma. World J Surg. 2005;29 (10):1268-1276. doi:10.1007/s00268-005-7745-9

19. Matthey-Gie ML, Gie O, Deretti S, Demartines N, Matter M. Prospective randomized study to compare lymphocele and lymphorrhea control following inguinal and axillary therapeutic lymph node dissection with or without the use of an ultrasonic scalpel. Ann Surg Oncol. 2016;23(5):1716-1720. doi:10.1245/s10434-015-5025-y

20. Liu CE, Lu Y, Yao DS. Feasibility and safety of video endoscopic inguinal lymphadenectomy in vulvar cancer: a systematic review. PLoS One. 2015;10(10):e0140873. doi:10.1371/journal.pone.0140873

21. Morotti M, Menada MV, Boccardo F, et al. Lymphedema microsurgical preventive healing approach for primary prevention of lower limb lymphedema after inguinofemoral lymphadenectomy for vulvar cancer. Int J Gynecol Cancer. 2013;23(4):769-774. doi:10.1097/ IGC.0b013e318287a8e8

22. Boccardo F, Valenzano M, Costantini S, et al. LYMPHA technique to prevent secondary lower limb lymphedema. Ann Surg Oncol. 2016;23(11):3558-3563. doi:10.1245/s10434-016-5282-4

23. Ozmen T, Lazaro M, Zhou Y, Vinyard A, Avisar E. Evaluation of Simplified Lymphatic Microsurgical Preventing Healing Approach (S-LYMPHA) for the prevention of breast cancer-related clinical lymphedema after axillary lymph node dissection. Ann Surg. 2018. doi:10.1097/SLA.0000000000002827

24. Spotnitz WD. Commercial fibrin sealants in surgical care. Am J Surg. 2001;182(2 Suppl):8S-14S.

25. Jackson MR. New and potential uses of fibrin sealants as an adjunct to surgical hemostasis. Am J Surg. 2001;182(2 Suppl):36S-39S.

26. Edwards SJ, Crawford F, van Velthoven MH, et al. The use of fibrin sealant during non-emergency surgery: a systematic review of evidence of benefits and harms. Health Technol Assess. 2016;20 (94):1-224. doi:10.3310/hta20940

27. Kawamura M, Sawafuji M, Watanabe M, Horinouchi H, Kobayashi K. Frequency of transmission of human parvovirus B19 infection by fibrin sealant used during thoracic surgery. Ann Thorac Surg. 2002;73(4):1098-1100.

28. Cipolla C, Fricano S, Vieni S, et al. Does the use of fibrin glue prevent seroma formation after axillary lymphadenectomy for breast cancer? A prospective randomized trial in 159 patients. J Surg Oncol. 2010;101(7):600-603. doi:10.1002/jso.21531

29. Docimo G, Limongelli P, Conzo G, et al. Axillary lymphadenectomy for breast cancer in elderly patients and fibrin glue. BMC Surg. 2013;13(Suppl 2):S8. doi:10.1186/1471-2482-13-S2-S8

30. Barranger E, Bricou A. Response to the article by Rodriguez et al: "effectiveness of an absorbable fibrin sealant patch to reduce lymphoceles formation after axillary lymphadenectomy for breast cancer: a matched-pair analysis." Am J Surg 2014. Am J Surg. 2015;209 (2):426-427. doi:10.1016/j.amjsurg.2014.06.028 
31. Navarro-Rodriguez E, Gomez-Luque I, Diaz-Jimenez N, et al. Effectiveness of an absorbable fibrin sealant patch to reduce lymphoceles formation after axillary lymphadenectomy for breast cancer: a matched-pair analysis. Am J Surg. 2014;208(5):824-830. doi:10.1016/j.amjsurg.2013.12.041

32. Sajid MS, Hutson KH, Rapisarda IF, Bonomi R. Fibrin glue instillation under skin flaps to prevent seroma-related morbidity following breast and axillary surgery. Cochrane Database Syst Rev. 2013;(5):CD009557.

33. Buda A, Ghelardi A, Fruscio R, et al. The contribution of a collagen-fibrin patch (Tachosil) to prevent the postoperative lymphatic complications after groin lymphadenectomy: a double institution observational study. Eur J Obstet Gynecol Reprod Biol. 2016;197:156-158. doi:10.1016/j.ejogrb.2015.12.005
34. Weldrick C, Bashar K, O'Sullivan TA, et al. A comparison of fibrin sealant versus standard closure in the reduction of postoperative morbidity after groin dissection: A systematic review and meta-analysis. Eur J Surg Oncol. 2014;40(11):1391-1398. doi:10.1016/j.ejso.2014.07.034

35. Neuss H, Raue W, Koplin G, Schwenk W, Reetz C, Mall JW. A prospective randomized trial: the influence of intraoperative application of fibrin glue after radical inguinal/iliacal lymph node dissection on postoperative morbidity. Eur J Surg Oncol. 2009;35 (8):884-889. doi:10.1016/j.ejso.2008.09.016

36. Diehl A, Volland R, Kirn V, et al. The number of removed lymph nodes by inguinofemoral lymphadenectomy: impact on recurrence rates in patients with vulva carcinoma. Arch Gynecol Obstet. 2016;294(1):131-136. doi:10.1007/s00404-015-3932-6

\section{Publish your work in this journal}

Cancer Management and Research is an international, peer-reviewed open access journal focusing on cancer research and the optimal use of preventative and integrated treatment interventions to achieve improved outcomes, enhanced survival and quality of life for the cancer patient.
The manuscript management system is completely online and includes a very quick and fair peer-review system, which is all easy to use. Visit http://www.dovepress.com/testimonials.php to read real quotes from published authors. 